Jannis Hagenah*, Sascha Leymann, and Floris Ernst

\title{
Integrating Label Uncertainty in Ultrasound Image Classification using Weighted Support Vector Machines
}

https://doi.org/10.1515/cdbme-2019-0072

\begin{abstract}
Inference from medical image data using machine learning still suffers from the disregard of label uncertainty. Usually, medical images are labeled by multiple experts. However, the uncertainty of this training data, assessible as the unity of opinions of observers, is neglected as training is commonly performed on binary decision labels. In this work, we present a novel method to incorporate this label uncertainty into the learning problem using weighted Support Vector Machines (wSVM). The idea is to assign an uncertainty score to each data point. The score is between 0 and 1 and is calculated based on the unity of opinions of all observers, where $u=1$ if all observers have the same opinion and $u=0$ if the observers opinions are exactly 50/50, with linear interpolation in between. This score is integrated in the Support Vector Machine (SVM) optimization as a weighting of errors made for the corresponding data point. For evaluation, we asked 15 observers to label 48 2D ultrasound images of aortic roots addressing whether the images show a healthy or a pathologically dilated anatomy, where the ground truth was known. As the observers were not trained experts, a high diversity of opinions was present in the data set. We performed image classification using both approaches, i.e. classical SVM and wSVM with integrated uncertainty weighting, utilizing 10-fold Cross Validation, respectively (linear kernel, $C=7$ ). By incorporating the observer uncertainty, the classification accuracy could be improved by 3.1 percentage points (SVM: $83.5 \%$, wSVM: $86.6 \%$ ). This indicates that integrating information on the observers' unity of opinions increases the generalization performance of the classifier and that uncertainty weighted wSVM could present a promising method for machine learning in the medical domain.
\end{abstract}

Keywords: machine learning, label uncertainty, image processing, ultrasound, classification

\footnotetext{
*Corresponding author: Jannis Hagenah, Sascha Leymann, Floris Ernst, Institute for Robotics and Cognitive Systems, University of Lübeck, Ratzeburger Allee 160, 23562 Lübeck, Germany, e-mail: hagenah@rob.uni-luebeck.de
}

\section{Introduction}

During the last years, machine learning gained attention in the medical domain as a powerful tool for computer assistance in medicine and healthcare. Especially in the field of medical image analysis, machine learning delivered promising results [1]. One application is the classification of images, for example to discriminate between healthy and pathological anatomies. Usually, supervised learning is applied. In this case, a human observer annotates each image by assigning a specific class label to it, for example healthy or ill. From this data set, the learning algorithm tries to extract patterns from the images to reproduce the observers decision.

However, medical image data sets usually suffer from a high inter-patient variability and the identification or categorization of pathological changes might be hard. Hence, if multiple observers are asked to annotate a data set, it is very likely that the observers do not always share the same opinion. Even though this information on the unity of opinion between the observers is assessible and might be highly relevant to increase the generalization performance of the learning algorithm, usually a majority vote is used to generate binary labels, i.e. the image is assigned to the class that most observers chose [1]. By applying this majority vote, all information on the interobserver variance is ignored. In this work, we present an approach to integrate the information on the observer's unity of opinion (OUO) into Support Vector Machine (SVM) classifiers for ultrasound image classification. Thus we define an image-dependent weighting factor based on the OUO and use this factor in a weighted Support Vector Machine (wSVM) setting, aiming on increasing the generalization performance of the classifier.

The contribution of this work is twofold. First, we present a data set of ultrasound images labeled by 15 observers that can serve as a benchmark data set for integrating label uncertainty in medical image classification. Second, to the best of our knowledge, we describe the first approach to integrate the OUO into ultrasound image classification using wSVMs. 


\section{Material and Methods}

Our methods section is divided into three parts. First, the underlying data set as well as the design of the observer labeling study is described. Afterwards, we present an explanation of our developed learning method, followed by details on our evaluation experiments.

\subsection{Dataset and Study Design}

In this initial study, we focussed on the discrimination of healthy and pathologically dilated aortic roots in ultrasound images. The dataset was published in [2] and consists of 2D ultrasound images of the commissure plane of 25 porcine aortic valves. The imaging was performed ex-vivo under physiologically realistic pressure. During the collection of this dataset, healthy aortic roots were imaged and then manipulated, i.e. artificially enlarged by sewing in additional tissue, to simulate a pathological dilation, followed by a second imaging. The imaging setup aims on mimicking a realistic transesophageal ultrasound examination. Details on the data collection procedure can be found in [2]. Therefore, the dataset consists of 50 images, where 25 images are healthy and 25 images are ill, respectively. Fig. 1 shows example images from the dataset.

Hence, the ground truth of the labels (healthy or ill) is known and the classes are perfectly balanced, which is highly relevant for the evaluation. Additionally, this discrimination is possible but quite challenging for untrained observers, resulting in .

We asked 15 untrained observers to assign the binary label to the images in our data set. Thus, we first introduced the medical background and showed one representative example image of each class. Afterwards, the probands labeled all of the 48 remaining images. As the labeling of the different observers was performed independently, we do not expect any biased correlation in the resulting decisions. The result of this study are 15 binary observer opinions for each of the $48 \mathrm{im}$ ages.

\subsection{Observer's Unity of Opinions weighted SVM}

In classical Support Vector Machines (SVM), the aim is to minimize the following objective function under the given constraints:

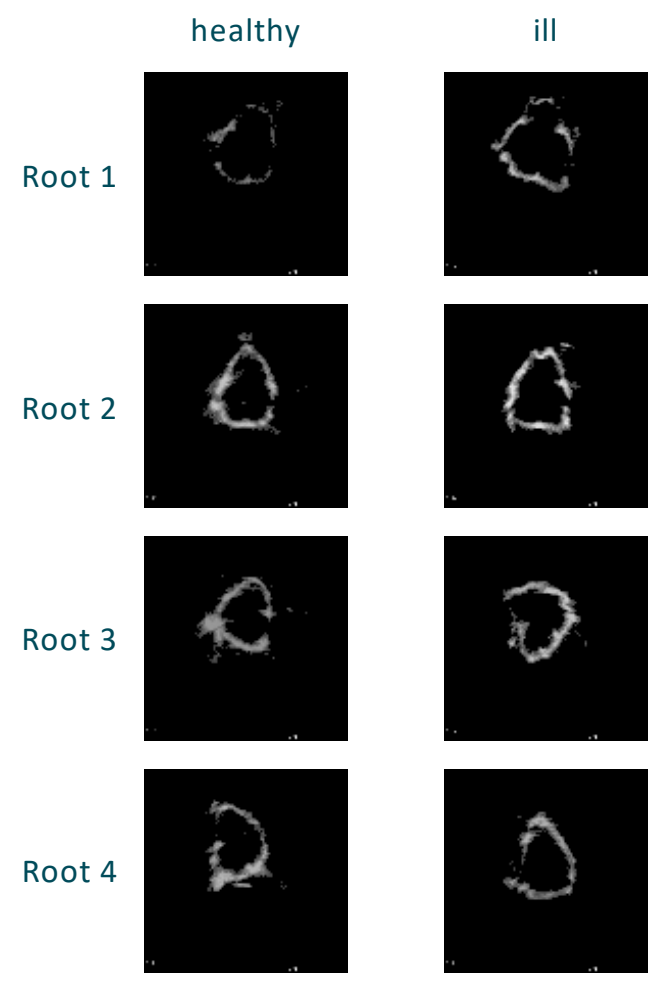

Fig. 1: Images from the data set, exemplarily shown for four aortic roots. In some cases, the dilation of the root is clearly visible (Root 1 and Root 2). In other cases, it is hard to identify the pathological changes (Root 3 and Root 4), resulting in a smaller OUO.

$$
\begin{array}{ll}
\underset{\mathbf{w}}{\operatorname{minimize}} & \frac{1}{2} \mathbf{w}^{T} \mathbf{w}+C \sum_{i=1}^{n} \xi_{i} \\
\text { subject to } & y_{i}\left(\mathbf{w}^{T} \Psi\left(x_{i}\right)+b\right) \geq 1-\xi_{i}, i=1, \ldots, n,
\end{array}
$$

where $\mathbf{w}$ and $b$ are parameters of the seperating hyperplane, $x_{i}$ and $y_{i}$ the $i$-th data point's features and label ( $y \in$ $\{-1,1\}), \Psi$ is a mapping to a space of higher dimension, usually induced by a Kernel, $\xi_{i}$ is the slack variable corresponding to the $i$-th data point, $C$ is a weighting parameter and $n$ is the number of data points [3]. Hence, the SVM optimizes to find the maxmimum margin seperating plane while all points are classified correctly, where outliers are allowed but penalized by the slack variables. The parameter $C$ controls the trade-off between a big margin and the number of wrongly classified data points.

The main idea of our work is to integrate the uncertainty given by the OUO into the training process. Thus, misclassifications of a data point should be penalized higher if the OUO is high. On the other hand side, a small OUO indicates a high uncertainty, and a misclassification at this data point should not be penalized harshly. Hence, we utilized the con- 
cept of weighted Support Vector Machines (wSVM) to weight the data points accordingly [4]. In comparison to a classical SVM, the wSVM introduces a new weighting factor $u_{i}$ for each data point:

$$
\begin{array}{ll}
\underset{\mathbf{w}}{\operatorname{minimize}} & \frac{1}{2} \mathbf{w}^{T} \mathbf{w}+C \sum_{i=1}^{n} u_{i} \xi_{i} \\
\text { subject to } & y_{i}\left(\mathbf{w}^{T} \Psi\left(x_{i}\right)+b\right) \geq 1-\xi_{i}, i=1, \ldots, m .
\end{array}
$$

This factor scales the penalization of classification errors observed at specific data points. Accordingly, the dual formulation of the wSVM is

$$
\begin{array}{ll}
\underset{\alpha}{\operatorname{maximize}} & \sum_{i=1}^{n} \alpha_{i}-\frac{1}{2} \sum_{i=1}^{n} \sum_{j=1}^{n} \alpha_{i} \alpha_{j} y_{i} y_{j} K\left(x_{i}, x_{j}\right) \\
\text { subject to } & \sum_{i=1}^{n} y_{i} \alpha_{i}=0 \\
& 0 \leq \alpha_{i} \leq u_{i} C, i=1, \ldots, m
\end{array}
$$

To integrate the OUO into the learning problem, we defined a function to derive the weighting factor $u$ from the annotations acquired during the study. The decision of the $k$-th observer on the $i$-th data point is given as $y_{i}^{k} \in\{-1,1\}, k=$ $1, \ldots, N$, where $N$ is the total number of observers. We define the weighting factor $u_{i}$ as

$$
u_{i}=\frac{1}{N}\left|s_{i}\right|
$$

with

$$
s_{i}=\sum_{k=1}^{N} y_{i}^{k} .
$$

As $y_{i} \in\{-1,1\}$, the sum $s_{i} \in[-N, N]$ over all decisions measures the tendency of the observers to vote for a specific class. Hence, $u_{i} \in[0,1]$ describes the unity of opinions of all observers for the $i$-th data point, where $u_{i}=1$ means that all observers share the same opinion. If $u_{i}=0$, the uncertainty is highest as the number of observers voting for each class is exactly identical. The function $u_{i}\left(s_{i}\right)$ is visualized in Fig. 2. Like this, we can derive $y_{i}$ with a majority vote decision and additionally integrate the uncertainty arising from the OUO by the weighting $u_{i}$. For convenience, $N$ should be an odd number to avoid random class selection during the majority vote process. As described above, $N=15$ for this specific study.

\subsection{Evaluation}

To evaluate our method, we trained the proposed wSVM as well as a classical SVM on the dataset to classify ultrasound

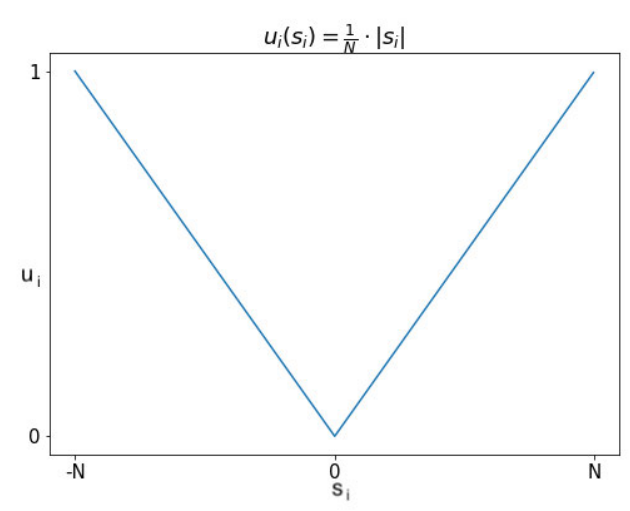

Fig. 2: The weighting parameter $u_{i}$ in dependency of the value of $s_{i}$. The higher the number of observers that share the same opinion, the higher is $u_{i}$.

images of the aortic valve as healthy or ill. For feature extraction, we used the convolutional layers of a pre-trained convolutional neural network ( 3 blocks of 2 convolutional layers followed by a max-pooling layer, respectively, 16 filters per layer, filter size $3 x 3$ ) that was trained on the same data set [5]. It is important to note that the aim of this study is not to find a suitable classifier for the given problem but to compare classical SVM with OUO weighted SVM for ultrasound image classification. Hence, the validation of this feature extraction is not in the scope of this work. Based on these features, we evaluated a classical SVM model as well as the wSVM model with OUO weighting using a 10-fold cross validation with random splits [6], where 33 images were in the training set and 15 images in the test set, respectively. For both models, we used a linear kernel and set $C=7$. The implementation was done using the scikit learn package [7]. In each run of the cross validation, we computed the prediction accuracy as the relative amount of test images being classified correctly.

\section{Results and Discussion}

During the study, we observed a high inter-observer variability in the annotations. The mean relative amount of observers that share the same opinion was $74.9 \pm 15.1 \%$, indicating that the task was quite challenging for the probands. This number highlights the importance of integrating this uncertainty information into the learning problem. The mean classification accuracy over all 10 runs was $83.5 \%$ for the classical SVM and $86.6 \%$ for the wSVM. Hence, by integrating the OUO into the learning problem, classical SVM could be outperformed and the classification accuracy could be increased by 3.1 percent- 
age points. This indicates that the wSVM model has a better capability of generalization for unseen data points.

Once again, it is important to notice that the aim of this study is not to solve the problem with the highest accuracy, but to use the OUO information in a multi-observer dataset and compare it to a scenario where we throw away this information using a majority vote. Hence, the absolute accuracy is not relevant and we do not compare the results to an SVM trained on the ground truth labels as this data would never be present in a real world scenario.

We see two main limitations of this study. First, the data set is very small, especially for an image classification problem. Second, the problem seems to be quite challenging for observers as well as for the classification algorithm, resulting in a small OUO and small absolute prediction accuracies. Hence, a deeper analysis of our proposed method on a vast data set is necessary even though the observer study might get very time consuming. Additionally, further work will include an analysis of the influence of the wSVM hyperparameters, i.e. the kernel and the choice of $C$, on the generalization performance of our method. One more interesting question is whether a nonlinear definition of $u_{i}\left(s_{i}\right)$ might increase the accuracy even further as small inter-observer variances could be ignored in the weighting function. However, these first results indicate that the integration of the OUO into an SVM classifier increases its capability of generalizing knowledge to learn ultrasound image classification.

\section{Conclusion}

In this work, we presented an approach for integrating label uncertainty arising from multiple observers into ultrasound image classification with SVMs. Thus, we defined the observer's unity of opinion (OUO) as well as a weighting factor $u$ for each data point based on the OUO, aiming on the usage of wSVMs for the classification problem. To evaluate our method, we conducted a study for acquiring annotations of 15 observers for discriminating healthy from ill aortic roots in 2D ultrasound images. We could show that a high inter-observer variabilty was present in our data and that our method outperformed classical SVMs by incorporating this uncertainty. This initial study indicates the high potential of integrating the OUO into machine learning problems. The general concept of point weighting based on the OUO is not limited to SVMs but also applicable in other classifiers, for example artificial neural networks. Besides the general use of our method in studies with multiple observers, learning on "weak labels", i.e. annotations acquired from untrained observers, is a very interesting application, especially in a medical context where only few experts are available. Hence, this study presents an important step towards reliable machine learning in medicine.

Acknowledgment: The authors would like to thank Michael Scharfschwerdt from the Department of Cardiac Surgery, University Hospital Schleswig Holstein, Lübeck, Germany for his help providing the data set.

\section{Author Statement}

Research funding: The author state no funding involved. Conflict of interest: Authors state no conflict of interest. Informed consent: Informed consent has been obtained from all individuals included in this study. Ethical approval: The research related to human use complies with all the relevant national regulations, institutional policies and was performed in accordance with the tenets of the Helsinki Declaration, and has been approved by the authors' institutional review board or equivalent committee.

\section{References}

[1] Giger, M. L. (2018). Machine learning in medical imaging. Journal of the American College of Radiology, 15(3), 512520.

[2] Hagenah, J., Werrmann, E., Scharfschwerdt, M., Ernst, F., Metzner, C. (2016). Prediction of individual prosthesis size for valve-sparing aortic root reconstruction based on geometric features. In 2016 38th Annual International Conference of the IEEE Engineering in Medicine and Biology Society (EMBC) (pp. 3273-3276). IEEE.

[3] Burges, C. J. (1998). A tutorial on support vector machines for pattern recognition. Data mining and knowledge discovery, 2(2), 121-167.

[4] Huang, Y. M., Du, S. X. (2005, August). Weighted support vector machine for classification with uneven training class sizes. In 2005 International Conference on Machine Learning and Cybernetics (Vol. 7, pp. 4365-4369). IEEE.

[5] LeCun, Y., Bengio, Y., Hinton, G. (2015). Deep learning. nature, 521(7553), 436.

[6] Stone, M. (1974). Cross-validatory choice and assessment of statistical predictions. Journal of the Royal Statistical Society: Series B (Methodological), 36(2), 111-133.

[7] Pedregosa, F., Varoquaux, G., Gramfort, A., Michel, V., Thirion, B., Grisel, O., et al. (2011). Scikit-learn: Machine learning in Python. Journal of machine learning research, 12(Oct), 2825-2830. 